\title{
Correlação entre a citologia e a histologia nas lesões intra- oculares suspeitas de malignidade
}

\author{
Cytologic-histologic correlation in intraocular lesions with suspicion of malignancy
}

\author{
Zélia Maria da Silva Corrêa \\ Luciane Dreher Irion ${ }^{2}$ \\ James J. Augsburger ${ }^{3}$ \\ Susan Schneider ${ }^{4}$ \\ Clélia Maria Erwenne
}

Serviço de Oftalmologia da Santa Casa de Porto AlegreRS

Este trabalho faz parte da tese apresentada pela primeira autora para obtenção do título de Doutora em Oftalmologia.

${ }^{1}$ Responsável pelo Setor de Oncologia Oftalmológica do Serviço de Oftalmologia da Santa Casa de Porto Alegre-RS. Pós-graduanda (nível doutorado), Universidade Federal de São Paulo -UNIFESP.

${ }^{2}$ Mestre em Patologia pela Fundação Faculdade Federal de Ciências Médicas de Porto Alegre - FFFCMPA- RS Laboratório Patologistas Reunidos - Porto Alegre-RS; ${ }^{3}$ Chairman do Departamento de Oftalmologia e "Professor" de Oftalmologia da Universidade de Cincinnati, $\mathrm{OH}$, EUA.

${ }^{4}$ Chefe do laboratório de Patologia Ocular e "Professora Assistente" da Universidade de Cincinnati, OH, EUA

Responsável pelo Setor de Oncologia Oftalmológica do Serviço de Oftalmologia da Universidade Federal de São Paulo - UNIFESP. Doutora em Oftalmologia pela Universidade Federal de São Paulo - UNIFESP.

Endereço para correspondência: Av. Nilo Peçanha,

2421 - Porto Alegre (RS) CEP 91330-001

E-mail:zmcorrea@terra.com.br

Recebido para publicação em 22.05.2002 Aceito para publicação em 16.12.2002

\section{R E S U M O}

Objetivo: Correlacionar os achados citológicos da biópsia aspirativa com agulha fina (BAAF) de lesões intra-oculares suspeitas de malignidade com a histopatologia obtida quando o tratamento de escolha foi enucleação ou ressecção da lesão tumoral. Métodos: Análise retrospectiva de 51 pacientes submetidos à biópsia aspirativa com agulha fina com fins diagnósticos ou para correlação cito-histológica. Foram excluídos os casos com lesões não sólidas, tratamento conservador e biópsias guiadas por ultra-som. Após exclusões, 20 olhos de pacientes contendo lesões intra-oculares suspeitas de malignidade foram estudados, sendo 12 do sexo feminino, com idades entre 2 e 78 anos. Todas biópsias foram realizadas pela primeira autora sob observação direta (microscópio) ou indireta (oftalmoscopia binocular indireta). A rota escolhida foi transaquosa para os tumores de segmento anterior e transvítrea com acesso escleral para os tumores de segmento posterior, exceto os casos suspeitos de retinoblastoma, biopsiados com acesso pela periferia da córnea. Foram obtidas 2 amostras de áreas diferentes do tumor em todos os casos com agulha calibre 25. As amostras colhidas foram encaminhadas para processamento, fixação e coloração pelo método de Papanicolaou e hematoxilina-eosina. Os espécimens obtidos para histopatologia foram corados pela hematoxilina-eosina. Resultados: Três casos eram tumores de segmento anterior (íris)e 17 de segmento posterior, sendo 3 retinoblastomas. Nove pacientes foram submetidos à biópsia aspirativa com agulha fina com fins diagnósticos e 11 para correlação cito-histológica após enucleação. Somente dois casos apresentaram quantidade insuficiente de material para diagnóstico e posteriormente revelaram ser um granuloma e um melanoma maligno de coróide. Conclusões: A biópsia aspirativa com agulha fina parece ser um método diagnóstico confiável baseado na correlação cito-histológica neste grupo de pacientes.

Descrittores:Biópsiaporagulha;Olho/citologia;Neoplasiasdacoróide/patologia;Neoplasias uveais/patologia;Melanoma;Retinoblastoma

\section{N T R O D U Ç Ã O}

A precisão do diagnóstico clínico oftalmológico de lesões intra-oculares suspeitas de malignidade melhorou muito desde a popularização do uso da oftalmoscopia binocular indireta e o desenvolvimento e aprimoramento de exames complementares como a angiografia fluorescente, a ultra-sonografia ocular, a tomografia computadorizada e a ressonância magnética ${ }^{(1)}$. Apesar dos índices de erro diagnóstico terem diminuído consideravelmente nas mãos de oftalmologistas especializados em oncologia ocular ${ }^{(2)}$, ainda existem 
casos difíceis que requerem mais recursos em sua elucidação diagnóstica.

Os primeiros relatos sobre o uso da biópsia aspirativa com agulha fina (BAAF) em oftalmologia foram publicados há cerca de $50 \operatorname{anos}^{(3)}$, mas seu uso mais intenso se deu a partir de 1979 com a publicação do artigo de Jakobiec et al. ${ }^{(4)}$. Após uma série de publicações de Augsburger et al. ${ }^{(1,5-8)}$ a BAAF começou a ser amplamente discutida e usada em vários centros de especializados em oncologia oftalmológica.

A indicação precisa da BAAF é muito importante quando se considera este método diagnóstico, uma vez que a sua utilização ainda não está indicada como rotina em todos os casos de tumores intra-oculares ${ }^{(1)}$. A BAAF tem sido realizada em nosso serviço com a aprovação do Comitê de Ética e Pesquisa nos seguintes casos: 1- incerteza diagnóstica mesmo após todos os exames não-invasivos que se dispõe, 2- recusa do paciente em aceitar tratamento mediante o diagnóstico clínico de neoplasia maligna intra-ocular e 3- na suspeita de tumor metastático quando se desconhece a neoplasia primária ou o paciente não tem diagnóstico de doença metastática.

Este estudo tem como objetivo correlacionar os achados citológicos da biópsia aspirativa com agulha fina de lesões intra-oculares suspeitas de malignidade com a histopatologia obtida quando o tratamento de escolha foi enucleação ou ressecção da lesão tumoral.

\section{MÉ TODOS}

Análise retrospectiva dos prontuários de 51 pacientes submetidos à biópsia aspirativa com agulha fina (BAAF) com fins diagnósticos ou para correlação cito-histológica durante o período de janeiro de 1997 a dezembro de 2001. Foram excluídos os casos de lesões não sólidas, biópsias guiadas pelo ultra-som devido à impossibilidade de visualizar o fundo de olho e aqueles tratados de forma conservadora o que impede a correlação histológica. Após exclusões, 20 olhos de pacientes contendo lesões intra-oculares suspeitas de malignidade foram estudados, sendo 12 do sexo feminino e 8 do masculino. A idade dos pacientes variou entre 2 e 78 anos (média $=46,25 \pm$ 24,23 anos). Todas as BAAF foram realizadas pela primeira autora após planejamento cuidadoso. Vários fatores foram considerados nesse planejamento: o diagnóstico presuntivo da massa intra-ocular, seu tamanho e localização, a presença e extensão do descolamento de retina e a transparência dos meios. Baseado nestas considerações, a via de entrada no globo ocular foi escolhida: pars plana para os tumores posteriores, exceto os retinoblastomas quando a entrada da agulha foi pela periferia da córnea através da raiz da íris, ou o limbo para os tumores do segmento anterior. As formas de monitorização do procedimento foram: direta (microscópio) ou indireta (oftalmoscopia binocular indireta) e o trajeto da agulha (trans-vítreo ou trans-aquoso), acompanhado através dos tecidos até sua introdução no tumor.

A técnica usada para a BAAF já foi previamente descrita ${ }^{(1,5-7,9)}$ Todos procedimentos foram realizados em ambiente cirúrgico, mesmo aqueles em olhos enucleados. Os casos de biópsia após enucleação (Figura 1), a BAAF foi realizada imediatamente após o procedimento cirúrgico com o objetivo de treinar a técnica de biópsia e estabelecer correlação cito-histológica, importante na experiência do citopatologista já que as amostras obtidas são sempre muito exíguas.

Nos casos de biópsia diagnóstica, o paciente foi colocado em decúbito dorsal horizontal sob bloqueio anestésico peribulbar com sedação leve. Os cuidados de assepsia foram semelhantes àqueles usados rotineiramente em cirurgias intraoculares. A agulha usada em todos os casos selecionados foi calibre ("gauge") 25 e comprimento de $30 \mathrm{~mm}$ para tumores de segmento posterior e $15 \mathrm{~mm}$ para aqueles de segmento anterior. A agulha foi conectada a um segmento de equipo de soro com cerca de $35 \mathrm{~cm}$ que, por sua vez, estava conectado a uma seringa descartável de $10 \mathrm{ml}$, com a qual o auxiliar realizava a aspiração manual. Após abertura da conjuntiva, reparo dos músculos retos para fixação do globo ocular, a agulha foi introduzida no globo ocular, sob monitorização (direta ou indireta), seu bisel direcionado para o tumor que em seguida foi perfurado pela agulha (Figura 2). Quando o bisel da agulha estava dentro do conteúdo tumoral, o auxiliar procedia à aspiração manual do mesmo. Depois a agulha era retirada num movimento contínuo seguindo trajeto semelhante ao que a mesma foi introduzida no olho. Em seguida, o auxiliar aplicava uma suave compressão digital no local de entrada da agulha por cerca de 60 segundos visando aumentar a pressão intraocular e diminuir o sangramento vítreo.

A agulha era então desconectada do tubo de silicone e conectada a uma seringa descartável de $5 \mathrm{ml}$ usada para aspirar 1-2 ml de solução salina balanceada com objetivo de lavar o conteúdo celular contido no lúmen da agulha. O mesmo procedimento era repetido para colher material de outra região do tumor. Foram obtidas 2 amostras de áreas diferentes do tumor em todos os casos.

O material obtido nas amostras era identificado e encaminhado imediatamente para processamento no laboratório de patologia. Após cito-centrifugado das amostras, cerca de $70 \%$

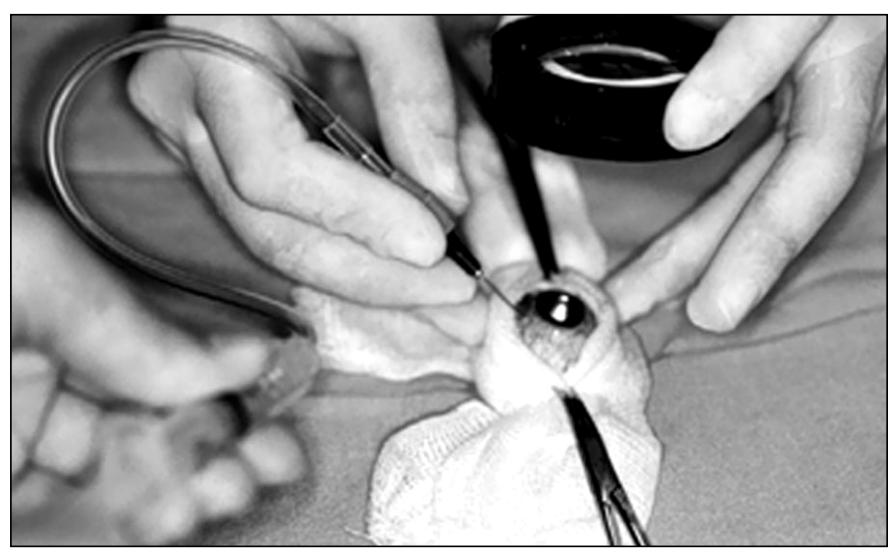

Figura 1 -Fotografia digital da técnica usada para biópsia aspirativa comagulhafina (BAAF)apósenucleaçãosobmonitorizaçãoindiretaem casos detumores de segmento posterior. Observe a mãodoauxiliar segurandoaseringa que faza aspiraçãomanual doconteúdotumoral 


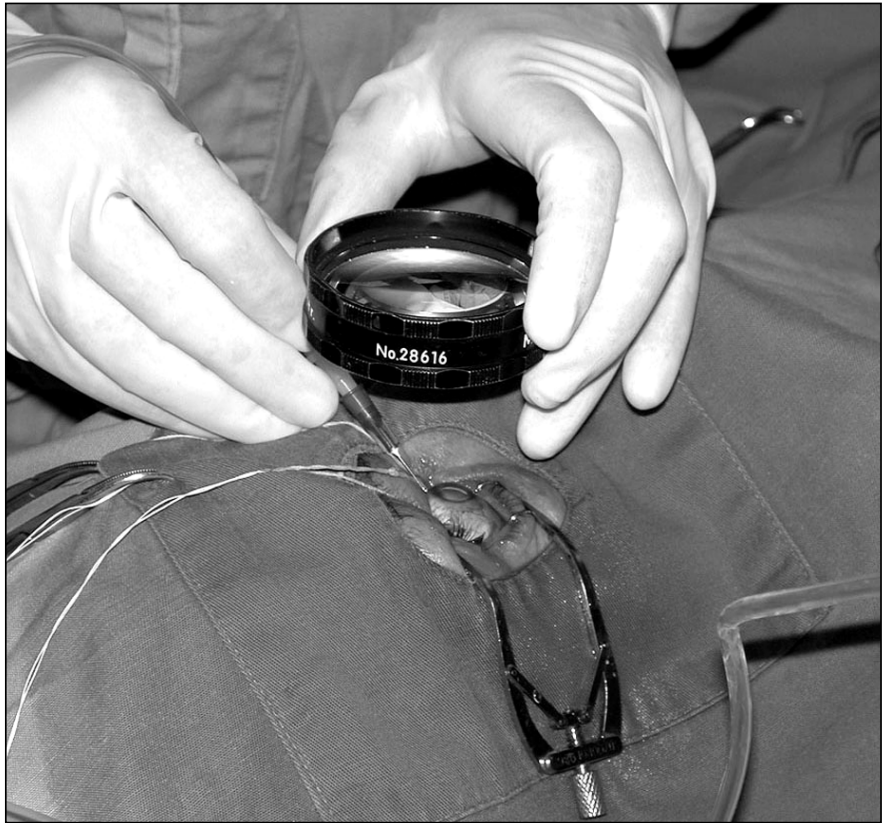

Figura 2-Fotografia digital da técnica usada para BAAF diagnóstica sobmonitorizaçãoindiretaemtumores desegmentoposterior(exceto casos suspeitos de retinoblastoma)

do material era imediatamente fixado em várias lâminas com álcool a 95\% e corado com os métodos: Papanicolaou e hematoxilina-eosina (HE). O restante do material era reservado para ser usado se a amostra fosse insuficiente ou para fazer um emblocado celular com parafina, corado com o método HE. Quando havia suspeita clínica de lesão metastática intra-ocular, o esfregaço era realizado em lâminas preparadas com organosilano, produto que contém um tipo de cola, possibilitando usar as mesmas lâminas para um eventual exame imunocitoquímico sem perda da amostra. Os espécimens obtidos por enucleação ou ressecção tumoral em bloco, foram encaminhados para histopatologia, fixados em formol a $20 \%$, processados em bloco de parafina para cortes histológicos e corados com método HE.

\section{RES ULT A D O S}

Dezessete casos eram tumores de segmento posterior sendo 3 retinoblastomas e os demais $(n=3)$ de segmento anterior (íris). Nove pacientes foram submetidos a BAAF com fins diagnósticos $(45 \%)$ e 11 para correlação cito-histológica após enucleação (55\%) (tabela 1). Dezenove pacientes (95\%) apre- sentavam sintomas visuais decorrentes da lesão intra-ocular em questão, entre eles um caso cujo material colhido na BAAF foi insuficiente para o diagnóstico. O período médio referido de sintomas visuais na data da consulta foi 4,97 meses. Somente dois casos (10\%) apresentaram quantidade insuficiente de material para diagnóstico e posteriormente revelaram ser um granuloma e melanoma maligno de coróide. A BAAF foi eficiente em diagnosticar 13 melanomas primários da coróide, sendo 2 de íris, 3 retinoblastomas, 1 linfoma e 1 granuloma fúngico. A correlação cito-histológica foi possível em 18 casos (90\%) (Figura 3). Entretanto, se forem considerados somente os casos com material suficiente para diagnóstico, a correlação cito-histológica foi $100 \%$ nesta série de casos. A histopatologia neste grupo de pacientes revelou 14 melanomas primários da coróide, 3 retinoblastomas, 1 linfoma e 2 granulomas (1 em retina, de etiologia fúngica e outro em íris).

A principal complicação observada no grupo de pacientes submetido à BAAF diagnóstica $(\mathrm{n}=9)$ foi algum grau de hemorragia intra-ocular no momento da retirada da agulha: hemorragia vítrea leve em 7 casos (Figuras 4 e 5), hemovítreo em 1 caso e hifema total em 1 caso. Não foi observada semeadura tumoral e/ ou delineação do trajeto da agulha no histopatológico dos casos estudados.

Até o presente momento, 18 pacientes estão vivos e sem sinais de doença metastática. Dois pacientes foram a óbito, ambos por doença metastática, 5 e 16 meses após BAAF, sendo uma diagnóstica e a outra após enucleação.

O tempo de seguimento destes pacientes variou entre 3-36 meses (média $=7,63$ meses).

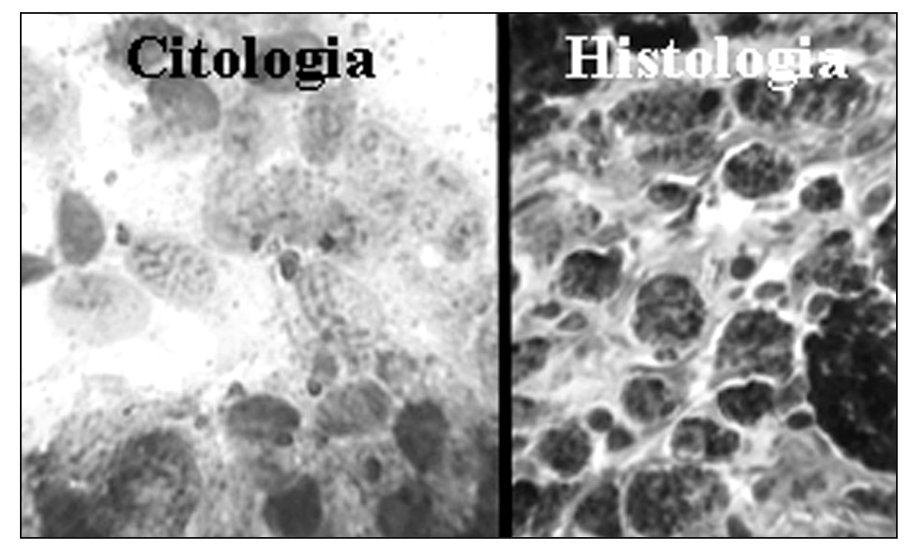

Figura 3-Fotografia digitalizada da citologia (coloracão commétodo de Papanicolaou)de um casode melanoma de conóidedotipomisto ea histologia do mesmo tumor, coradocom HE

\begin{tabular}{|c|c|c|c|c|}
\hline \multirow[b]{2}{*}{ Tipo de Biópsia (BAAF) } & \multicolumn{4}{|c|}{ Diagnósticocitológico } \\
\hline & Necasos & Maligno & Benigno & QID \\
\hline Pós-cinúrgicas (correlaçãocito-histológica) & 11 & 10 & & 1 \\
\hline Diagnósticas (pré-tratamento) & 9 & 7 & 1 & 1 \\
\hline
\end{tabular}




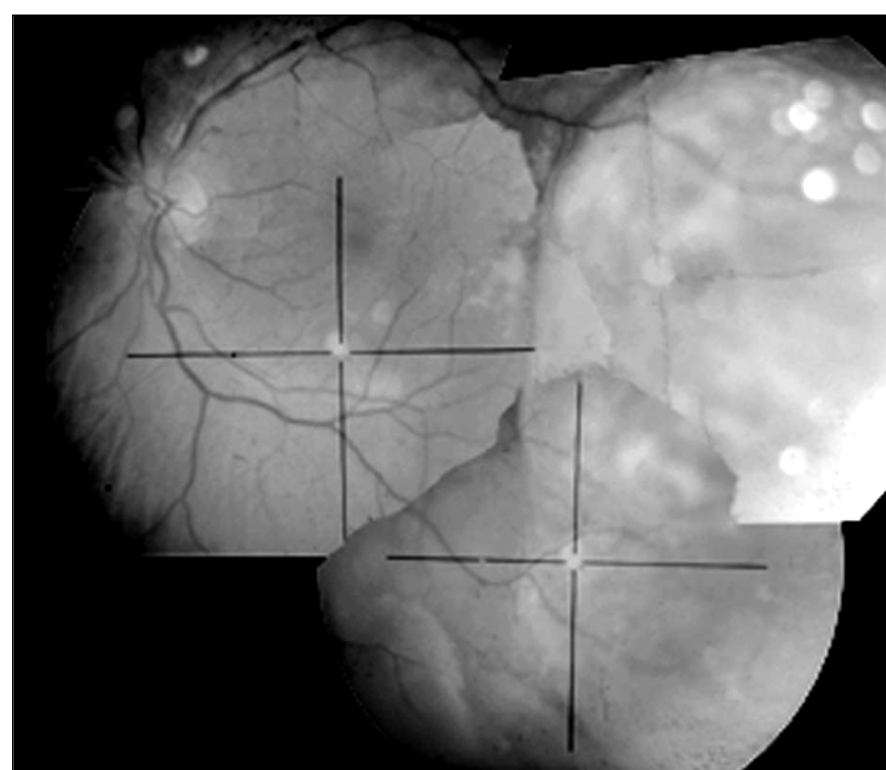

Figura4-Fotografiacompostadigitalmentedoaspectofundoscópicode umalesãosólidasuspeitademalignidade (melanomaversus metástase) em paciente do sexo feminino, 59 anos, com história pregressa de nódulode mama.A paciente foisubmetidaà BAAF diagnóstica antes dotratamento

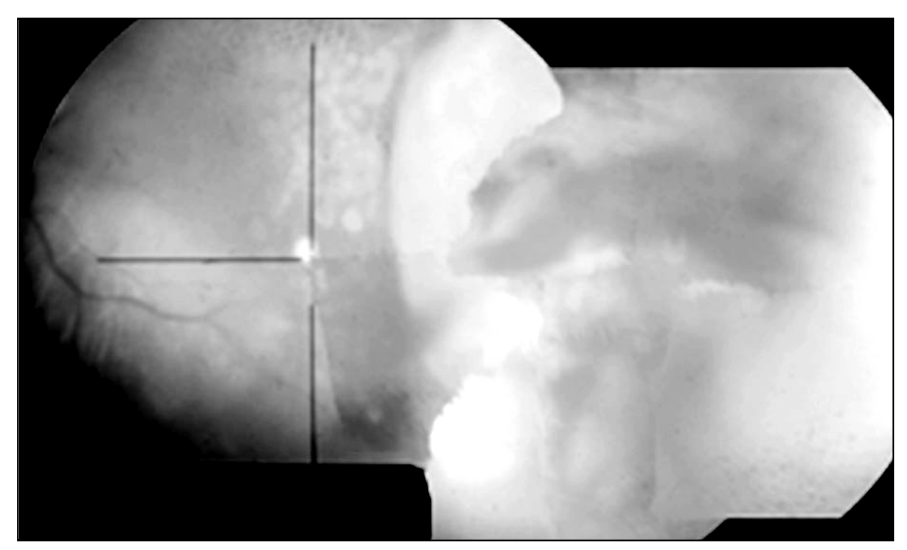

Figura 5-Fotografia compostadigitalmentedoaspectofundoscópico damesma lesão acima isemanaapós BAAF. Observea presença de hemorragia vítrea leve sobre a lesão e ausência de semeadura tumoral aparente. Este caso foi diagnosticado pela citologia como melanomaamelanóticoprimárioda coróide

D I S C US S ÃO

A biópsia aspirativa com agulha fina (BAAF) não é um procedimento usado com freqüência em nosso meio e as publicações internacionais sobre este procedimento parecem estar limitadas a certos grupos que se dedicam a aprimorar esta técnica ${ }^{(1,3,5-8)}$. A experiência inicial com esta técnica pode ser dificultada pelo uso da monitorização pela oftalmoscopia indireta, daí a importância de biopsiar repetidas vezes todos os olhos submetidos a enucleação. Este treinamento, além de aprimorar a técnica do cirurgião é muito importante para estabelecer padrões de diagnóstico para o citopatologista que precisará lidar com amostras diminutas de material ocular. Estes procedimentos, sugeridos em publicações por Augsburger e colaboradores ${ }^{(1,5-8)}$, têm servido como amadurecimento de toda a equipe multidisciplinar envolvida no diagnóstico e tratamento de tumores oculares.

A técnica usada neste grupo de pacientes foi amplamente discutida na comunidade oftalmológica que se dedica à oncologia ${ }^{(1,5-11)}$. Vários artigos ressaltando a importância e os problemas de basear o diagnóstico de uma lesão intra-ocular foram publicados ${ }^{(12)}$. As complicações, geralmente leves como a hemorragia vítrea, costumam se resolver sem nova intervenção ${ }^{(7-9)}$. Entretanto devem ser lembradas as possibilidades de ocorrerem descolamentos de retina, perfuração da parede ocular posterior, catarata traumática e até disseminação tumoral ${ }^{(5-7,12)}$.

Neste grupo de pacientes, apesar de pequeno, não ocorreram complicações além do sangramento intra-ocular limitado. Não foi observado trajeto de células tumorais no vítreo ou câmara anterior e, naqueles olhos enucleados em outro procedimento cirúrgico, não foi detectada presença de semeadura tumoral intra-ocular. Os índices relatados de complicações decorrentes da BAAF seriam em torno de 2-5\%(5-7). Entretanto, devemos nos ater ao problema dos retinoblastomas que são tumores muito friáveis com forte tendência à implantação tumoral. Uma série de 77 casos de diferentes tumores pediátricos previamente publicada relatou 38 casos de retinoblastomas submetidos à $\mathrm{BAAF}^{(13)}$. A correlação cito-histológica nesta série de casos foi $95 \%$, entretanto a maioria dos retinoblastomas foi biopsiada após enucleação devido às características tumorais descritas acima ${ }^{(13)}$. Os retinoblastomas devem ser biopsiados através da córnea, atravessando a base da íris e zônula, para evitar a disseminação sub-conjuntival deste tumor ${ }^{(13)}$. Em nossa série, somente um caso de retinoblastoma foi submetido a BAAF diagnóstica com o compromisso dos pais de autorizarem a enucleação imediata caso o diagnóstico fosse confirmado. Esta biópsia diagnóstica foi realizada há 14 meses e a criança permanece bem, sem sinais de doença metastática ou comprometimento orbitário até o presente momento.

Outro fator importante é o manejo dos casos de quantidade insuficiente para diagnóstico citológico ${ }^{(14)}$. Este é outra controvérsia na BAAF porque o diagnóstico inconclusivo seria considerado uma falha neste procedimento. Schneider e colaboradores abordaram este tema e concluíram que os tumores com características celulares mais agressivas tendem a ser mais discoesos e friáveis, portanto seria mais fácil colher material nestas circunstâncias ${ }^{(14)}$. Nesta série, esta hipótese se confirmou porque o primeiro caso de quantidade insuficiente foi um granuloma e o outro foi um melanoma de coróide que havia sido detectado pela primeira vez há 2 anos, cujo tamanho e a presença de glaucoma neovascular indicaram uma enucleação. Entretanto, a histologia revelou a baixa malignidade deste tumor que era composto predominantemente por células fusiformes A e algumas, raras fusiformes B.

Entre os casos com quantidade suficiente de material, todos tiveram uma correlação cito-histológica positiva. Tal fato provavelmente se deve à amostra reduzida apesar da BAAF tem 
se mostrado um método diagnóstico de boa confiabilidade, geralmente superior a $90 \%^{(1,5-7,11,13,15)}$.

Atualmente o grande desafio é o diagnóstico precoce de lesões melanocíticas pequenas especialmente em segmento posterior (coróide). A BAAF provavelmente terá um papel importante no diagnóstico destas lesões que estão sendo tratadas cada vez mais precocemente com novas técnicas conservadoras como laser e radioterapia ${ }^{(15)}$. O diagnóstico precoce garantiria um tratamento menos agressivo e com maior chance de conservação da visão. Em nossa série, não foram incluídos casos de lesões com estas características uma vez que estas raramente são tratadas com enucleação.

A sobrevida destes pacientes deve ser discutida já que um dos maiores receios em relação a esta técnica diagnóstica é a disseminação tumoral sistêmica. Os 2 óbitos ocorridos não pareceram estar relacionados com o procedimento. Um deles foi o caso de linfoma que a paciente já apresentava várias metástases sistêmicas anterior a BAAF e a indicação de biópsia foi solicitação da paciente. Cinco meses após a BAAF, a paciente faleceu e a família autorizou a enucleação do olho para correlação cito-histológica. O outro caso foi um paciente com melanoma de coróide considerado grande e glaucoma secundário. A biópsia, neste caso, foi realizada após a enucleação portanto não podendo ser responsável pelo óbito ocorrido 16 meses depois. Os demais pacientes $(n=18)$ estão sendo acompanhados regularmente pelo oftalmologista e oncologista clínico, nos casos de neoplasia maligna, para pesquisa de metástases sistêmicas.

\section{O N C L U S Ã O}

A BAAF pareceu ser um método diagnóstico confiável baseado na correlação cito-histológica neste grupo de pacientes. Entretanto grupos maiores de pacientes serão necessários para que este procedimento diagnóstico seja adotado como rotina em olhos com lesões intra-oculares suspeitas de malignidade.

A B S T RA C T

Purpose: To correlate the cytologic findings of fine-needle aspiration biopsy (FNAB) of intraocular tumors with the histopathology specimen when enucleation or tumor resection was the treatment of choice. Methods: Retrospective analysis of 51 patients submitted to fine-needle aspiration biopsy for diagnostic purposes or cyto-histologic correlation. Exclusion criteria were non-solid lesions, conservative treatment and ultrasound guided biopsies. After exclusions, 20 patients with intraocular solid lesions suspected to be malignant were studied, 12 of them were females, with ages between 2 and 78 years. All biopsies were performed by the first author under direct (operating microscope) or indirect (binocular indirect ophthalmoscope) visualization. The chosen route was transaqueous for anterior segment tumors and transvitreous through the sclera for posterior segment tumors except cases with suspicion of retinoblastoma biopsied through the peripheral cornea. Two samples were obtained from different areas of the tumor, in all cases with a 25-gauge needle. After fine-needle aspiration biopsy, samples were sent for processing, fixation, and staining with the Papanicolaou and hematoxylin-eosin (HE) methods. Histology specimens were stained with hematoxylin-eosin. Results: Three cases consisted of anterior segment (iris) tumors and 17 of tumors situated in the posterior segment, 3 of them retinoblastomas. Nine patients were biopsied for diagnostic purposes and 11 for cytological-histological correlation after enucleation. In only two cases, fine-needle aspiration biopsy yielded insufficient material for diagnosis that subsequently proved to be a granuloma and a melanoma. Conclusions: Fine-needle aspiration biopsy seems to be a reliable procedure based on cytological-histological correlations in the studied group.

Keywords: Needle biopsy; Eye/cytology; Choroid neoplasms/ pathology; Uveal neoplasms/pathology; Melanoma; Retinoblastoma

REFEREN C I A S

1. Augsburger JJ, Shields JA, Folberg R, Lang W, O’Hara BJ, Claricci JD. Fine needle aspiration biopsy in the diagnosis of intraocular cancer. Cytologichistologic correlations. Ophthalmology 1985;92:39-49.

2. Accuracy of diagnosis of choroidal melanomas in the Collaborative Ocular Melanoma Study [published erratum in Arch Ophthalmol 1190;108:1708]. Arch Ophthalmol 1990;108:1268-73.

3. Veasey Jr. CA. Intraocular biopsy. Am J Ophthalmol 1951;34:432-4.

4. Jakobiec FA, Coleman DJ, Chattock A, Smith M. Ultrasonically guided needle biopsy and cytologic diagnosis of intraocular tumors. Ophthalmology 1979;86:1662-81.

5. Augsburger JJ, Shields JA. Fine needle aspiration biopsy of solid intraocular tumors: indications, instrumentation and techniques. Ophthalmic Surg 1984; 15:34-40.

6. Augsburger JJ, Shields JA, Lang W. Fine needle aspiration biopsy of intraocular tumors [abstract]. Invest Ophthalmol 1986;27(Suppl):260.

7. Augsburger JJ. Fine needle aspiration biopsy of suspected metastatic cancer to the posterior uvea. Trans Am Ophthalmol Soc 1988;86:499-560.

8. Augsburger JJ. Fine needle aspiration biopsy in the diagnosis of suspected intraocular cancer, In: Jakobiec FA, Sigelman J, editors. Advanced techniques in ocular surgery. Philadelphia: W.B.Saunders; 1984. p.341-58.

9. Corrêa ZMS, Marcon IM. O uso da biópsia aspirativa com agulha fina no diagnóstico de lesão iriana suspeita. Arq Bras Oftalmol 2001;64:347-50.

10. Ritland JS, Eide N, Hoie J. Fine-needle aspiration biopsy diagnosis of a uveal metastasis from a follicular thyroid carcinoma. Acta Ophthalmol Scand 1999; 77:594-6.

11. Eide N, Syrdalen P, Walaas L, Hagmar B. Fine-needle aspiration biopsy in selecting treatment for inconclusive intra-ocular disease. Acta Ophthalmol Scand 1999;77:448-52.

12. Foulds WS. The uses and limitations of intraocular biopsy. Eye 1992;6:11-27.

13. O'Hara BJ, Ehya H, Shields JA, Augsburger JJ, Shields CL, Eagle RC. Fine needle aspiration biopsy in pediatric ophthalmic tumors. Acta Cytol 1993; $37: 125-30$

14. Schneider S, Augsburger JJ, Ehya H. Fine needle aspiration biopsy of solid intraocular tumors: the implications of an insufficient aspirate for cytopathologic diagnosis [abstract]. Invest Ophthalmol Vis Sci 1999;40(Suppl 4):244.

15. Augsburger JJ, Schneider S, Ehya H. The role of transvitreal FNAB in suspected small choroidal melanomas [abstract]. Invest Ophthalmol Vis Sci 1999; 40(Suppl 4):2 44. 